\title{
An Enhanced Biometric System for Personal Authentication
}

\author{
Umamaheswari.R ${ }^{1}$, Geetharamani.J ${ }^{2}$ \\ ${ }^{1} P G$ Scholar, ECE Dept, SNS College of Technology,Coimbatore, India, \\ ${ }^{2}$ Assistant Professor, ECE Dept, SNS College of Technology, Coimbatore, India,
}

\begin{abstract}
Palm vein authentication is a new and latest biometric method utilizing the vein patterns inside one's palm for personal identity verification. Palm patterns are different for each person.and as they are hidden underneath the skin's surface,forgery is extremely difficult.Infrared light is used to capture an image of a palm that shows the vein patterns, which have various widths and brightness that change temporally as a result of fluctuations in the amount of blood in the vein,depending on temperature,physical conditions,etc.To robustly extract the precise details of the depicted veins, we developed a method of Anisotropic technique in crosssectional profiles of a vein image.This method can extract the centrelines of the veins consistently without being affected by the fluctuations in vein width and brightness, so its pattern matching is highly accurate. This paper discusses the origins, feature extraction, technology, applications of palm vein authentication. The proposed system include: 1) Infrared palm images capture; 2) Detection of Region of Interest; 3) Palm vein extraction by Anisotropic filtering; 4) Matching. The experimental results demonstrate that the recognition rate using palm vein is good.
\end{abstract}

Index Terms-Palm vein, Liveness detection, Infrared palm images, Anisotropic filtering.

\section{INTRODUCTION}

The personal identification using hand and palm vein has gained more and more research attentions these years [1] [2] [3] [4] [5]. There are many good properties of this kind of biometric feature: 1) the vein information can represent the liveness of an object; 2) it is difficult to be damaged and modified as an internal feature; 3) it is difficult to simulated using a fake palm. Because of these, hand and palm vein seems a better biometric feature that finger print and face.In [1] [2], the thermal images are used to extract palm vein and obtained good results. But the infrared thermal camera is very expensive. In [5], a low cost CCD camera is used to capture near infrared image of palm. That system's recognition rate is good. But because both vein and texture information are used in [5] for recognition, that method cannot ensure the liveness of a person.In this work, we use a low cost CCD camera to capture the infrared palm images. The palm vein, rather than hand vein (back of the hand), is used by our system because it is easier to design a platform to help a user fixing his/her hand on the image capture device. And, we only use the palm vein as biometric feature without any other features such as palm texture and palm line so that this system can ensure the liveness of an object.

\section{IMAGE CAPTURE AND ROI LOCATING}

The capture device is modified from our previous work on palm print [6]. Fig. 1 shows part of our device. There are three poles to help a user to fix his/her hand (Fig. 1(b)).A low cost CCD camera is used in this system. In order to obtain infrared images, a set of infrared light source is installed around the camera.

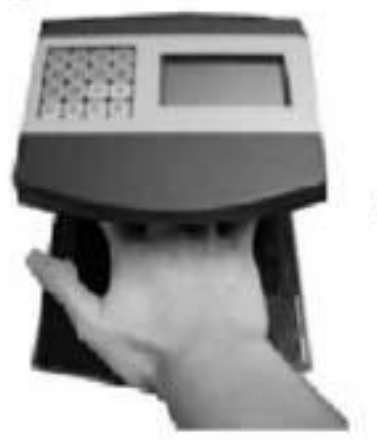

(a) 


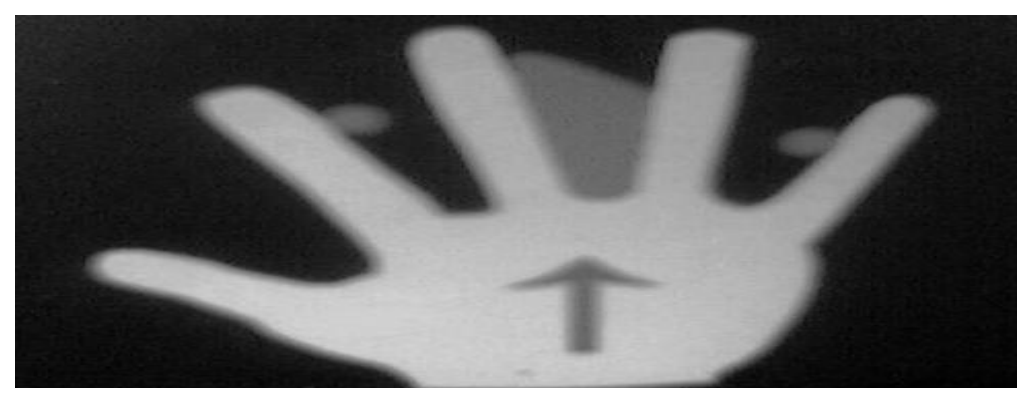

(b)

Fig. 1. Capture device. (a) outside of the device; (b) poles to fix a palm

After image capture, the region of interest (ROI) is located by the same method of our previous work [6]. A small area (128*128 pixels) of a palm image is located as ROI to extract the features and to compare different palms. Using the features within ROI for recognition can improve the computation

After image capture, the region of interest (ROI) is located by the same method of our previous work [6]. A small area (128*128 pixels) of a palm image is located as ROI to extract the features and to compare different palms. Using the features within ROI for recognition can improve the computation efficiency significantly. Further, because this ROI is located by a normalized coordinate based on the palm boundaries, the recognition error caused by a user who slightly rotate or shift his/her hand is minimized.

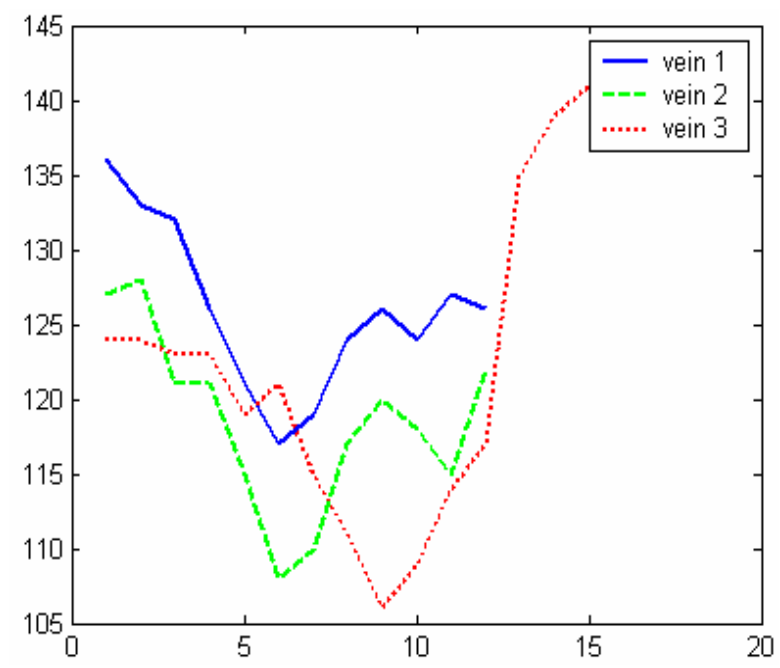

Fig. 2. Cross section evaluation

\section{Palm Vein Extraction}

By observing the cross-sections of palm veins, we found that they are Gaussian shaped lines. Fig. 2 shows some cross-sections of the palm veins. Based this observation, the Anisotropic filter proposed in [7] [8] can be used to detect palm veins. And, we propose a multiscale scheme to improve the performance of vein detection. This scheme includes multiscale Anisotropic filters and scale production [9] [10] [11].

The Anisotropic filter was defined as

$$
\left\{\begin{array}{c}
g_{\phi}(x, y)=-\exp \left(-x^{\prime 2} \mid \sigma_{x}^{2}\right)-m, \text { for } x^{\prime}\left|\leq 3 \sigma_{x}, \quad y^{\prime}\right| \leq L / 2 \\
x^{\prime}=x \cos \phi+y \sin \phi \\
y^{\prime}=y \cos \phi-x \sin \phi
\end{array}\right.
$$

This filter can be regarded as Gaussian filter in $\mathrm{x}$ direction. A Gaussian shaped filter can help to denoise and the zero-sum can help to suppress the background pixels. Fig. 3 shows the Anisotropic filters in 1-D (a) and 2-D (b) view. Fig. 4 gives the filter response in a single scale. 

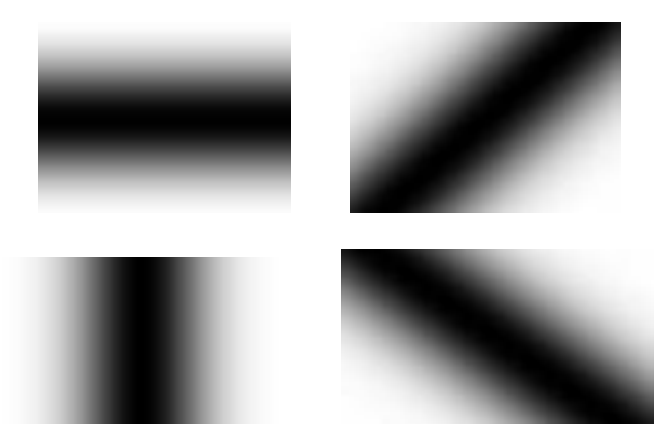

Fig. 3. Anisotropic filters (a) 1-D and (b) 2-D

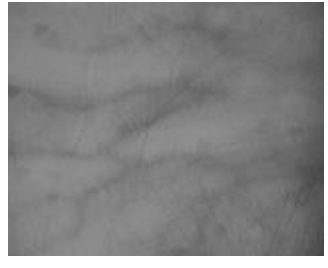

(a)

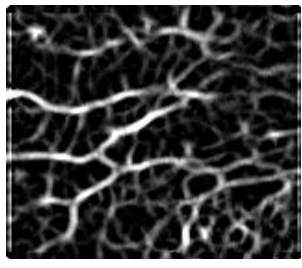

(b)

Fig. 4. (a) The subimage within ROI and (b) its single scale Anisotropic filter response

From Fig. 4, we find that there is too much noise in the anisotropic filter responses of infrared palm images. To gain a proper signal-noise ratio, we propose a multiscale scheme to detect the palm vein. In [12], Mallat illustrated mathematically that signals and noise have different singularities and that edge structures present observable magnitudes along the scales, while noise decreases rapidly. With this observation, we responded to those problems of edge and line detection and noise reduction by thresholding the multiscale products [9] [10] [11]. For Multiscale analysis, a scale parameter is added to equation (1) to control the filter size:

$$
g_{\phi, s}(x, y)=-\exp \left(-x^{\prime 2} / s \sigma_{x}^{2}\right)-m
$$

The response of multiscale Anisotropic filter can be expressed by

$$
R_{g}(x, y)=g_{\phi, s}(x, y) * f(x, y)
$$

The scale production is defined as the product of filter responses at two adjacent scales

$$
P^{s_{j}}(x, y)=R_{g}^{s_{j}}(x, y) \cdot R_{g}^{s_{j+1}}(x, y)
$$

Fig. 5 illustrates the multiscale line detection and scale production, where Mf1 and Mf 2 are the responses at two different scales, P1,2 is their production. The noise in Mf1 and Mf2 nearly reaches the half peak of the signal response. After production, the noise becomes much smaller. 


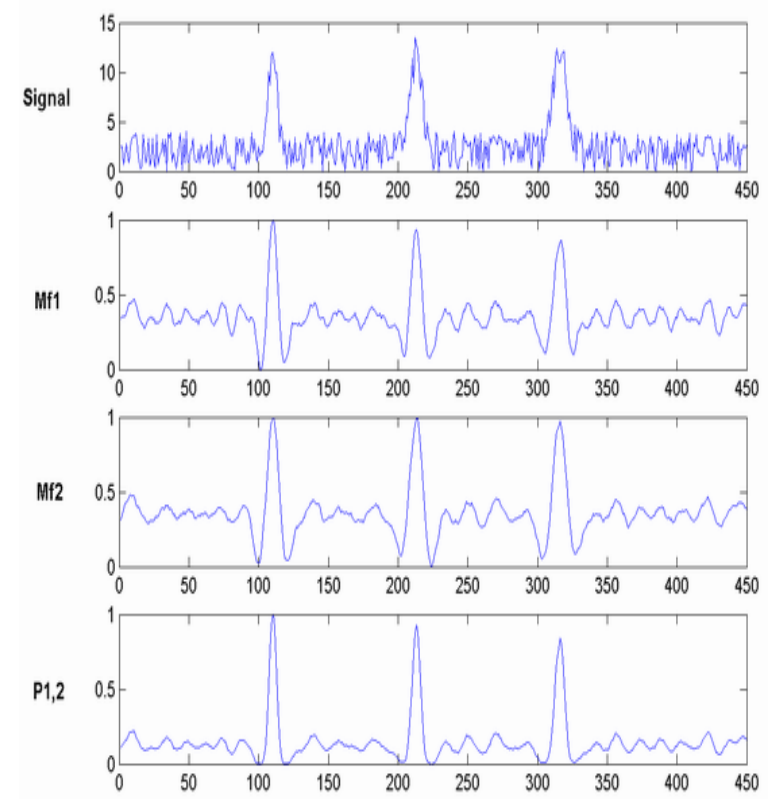

Fig. 5 Anisotropic filtering and scale production: Mf1 and Mf2 are the responses at two different scales, P1,P2 is their production According to our experience, the palm vein widths in our infrared images vary from 10 pixels to 20 pixels that corresponding to Gaussian with standard derivation from 1.2 to 2.4. In order to produce strong filter responses, the standard derivation of the anisotropic filters must be similar to the standard derivation of veins.

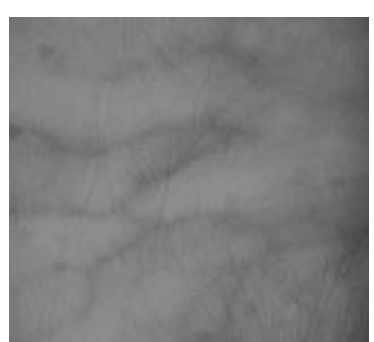

(a)

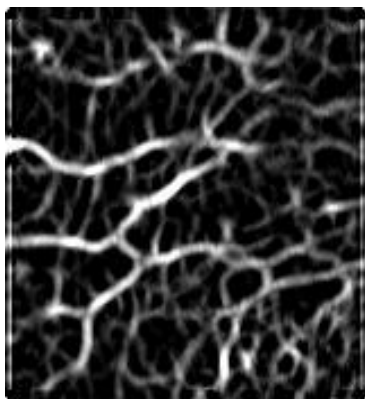

(b)

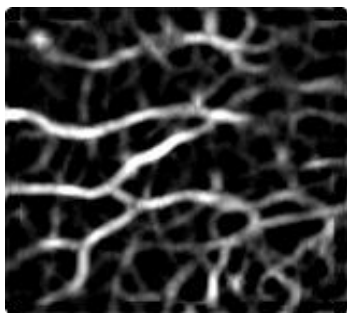

(c)

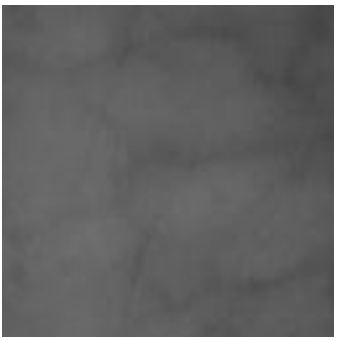

(f)

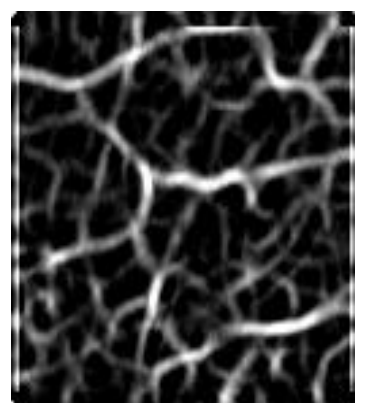

(g)

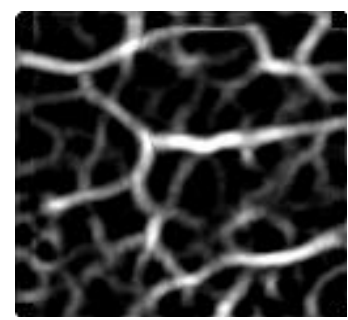

(h) 


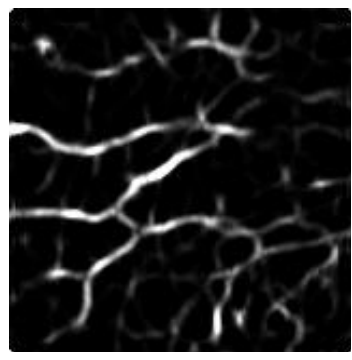

(d)

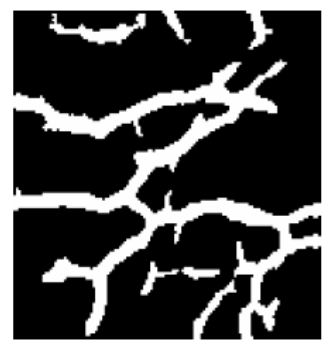

(e)

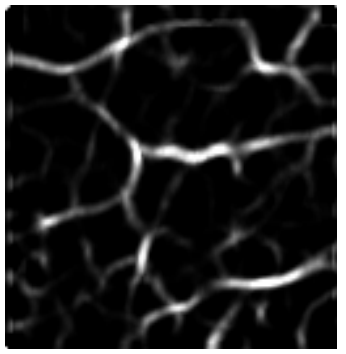

(i)

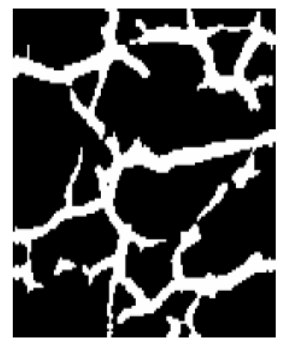

(j)

Fig. 6. Thresholding multiscale products: (a) a subimage, (b) \& (c) its Anisotropic filter responses at two different scales, (d) scale production of (b) \& (c), (e) binarized image; (f) (j) corresponding images of another palm.

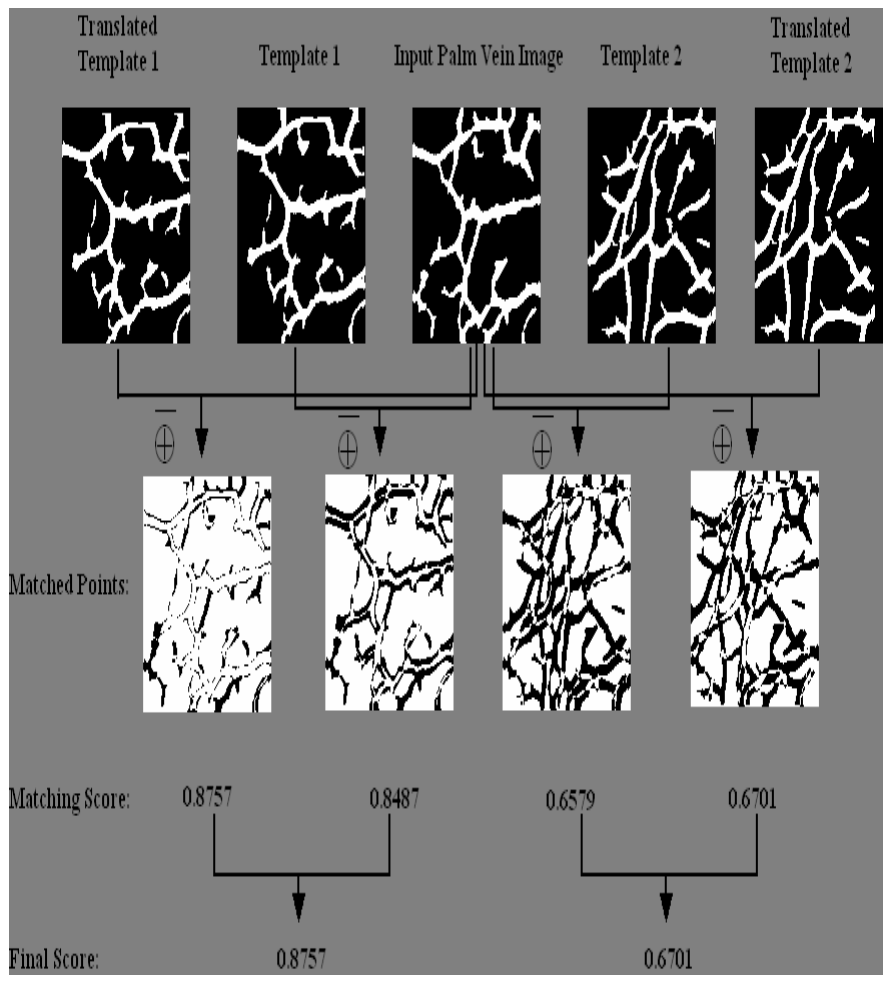

Fig. 7. Matching procedure

After multiscale filtering and scale production, the filter responses will be binarized by thresholding the multiscale products [9] [10]. Fig. 6 gives some examples of thresholding multiscale products of infrared palm images. Fig. 6(a) is a subimage of an infrared palm image within ROI; Fig. 6(b) and (c) are anisotropic filter responses at different scales; Fig. 6(d) is the scale production of (b) and (c); Fig. 6(e) is the binarized image of (d). Fig. 6(f) (i) are the corresponding images of another palm. The lines in the binarized images, which represent palm vein, are used as biometric features in our system. This binarized image is named as palm vein image in the following paper. 


\section{Palm Vein Matching}

Using the palm vein image as palm vein templates (only 0 and 1 in the templates), the similarity of two palm images can be calculated by template matching. Let $\mathrm{T}$ denote a prepared template in the database and $I$ denote the palm vein image of a new input palm, we match $T$ and $I$ through logical "exclusive or" operation. The matching scoreof $T$ and $I$ is calculated as

$$
S(T, I)=\frac{1}{M \times N} \sum_{i=1}^{M} \sum_{j=1}^{N}[\overline{T(i, j) \oplus I(i, j)}]
$$

Even we already registered palm images captured at different times at the step of ROI locating, there may still be little rotation and translation between them. To overcome this problem, we vertically and horizontally translate the template $T$ a few points. The final matching score is taken to be the maximum matching score of all the translated positions. This matching procedure is illustrated in Fig.7.

\section{Experimental Results}

There are totally 144 infrared palm images in our database. Each of 24 individuals has 6 images. Figure 8 shows images of a palm captured at different times, where the image quality is good. Figure 9 shows images of a palm captured at different times, where the image quality is bad.
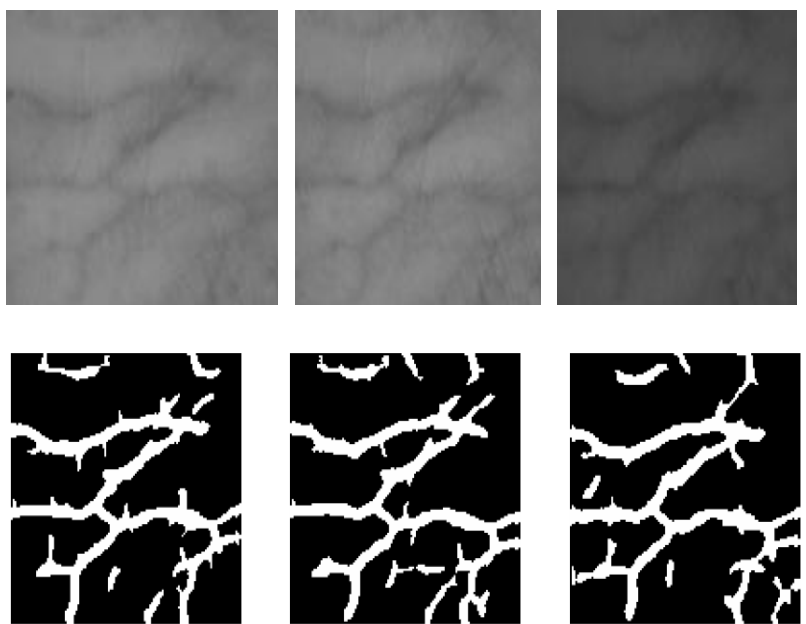

Fig. 8. Images of a palm captured at different times, where the image quality is good. The first row shows the images captured by our device. The second row shows the corresponding vessel extraction results. The recognition performance is shown in Figure 11 using ROC curve. We achieved 98.8\% recognition rate where the false acceptance rate is 5.5\%. Most of the false recognitions were caused by the images of poor quality.
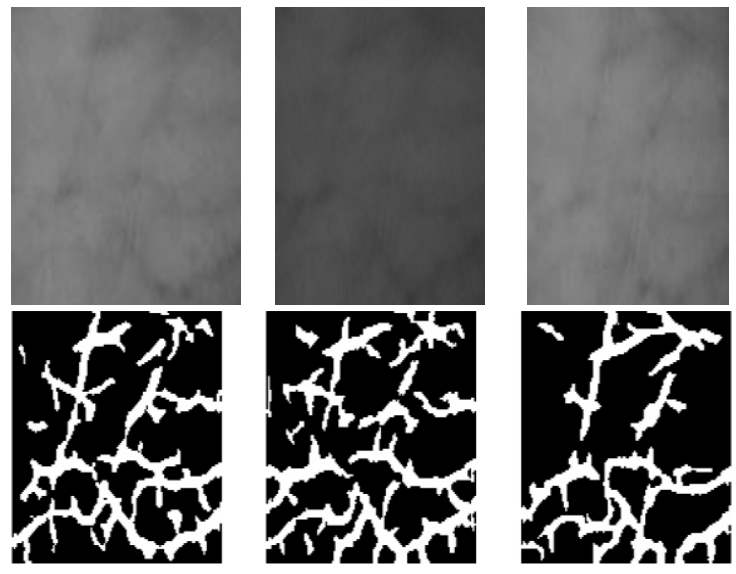

Fig. 9. Images of a palm captured at different times, where the image quality is bad. The first row shows the images captured by our device. The second row shows the corresponding vessel extraction results. 


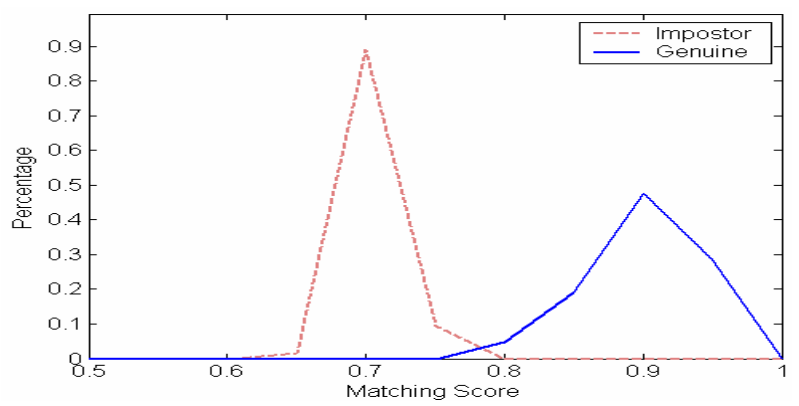

Fig. 10. The probability distribution of genuine and imposter

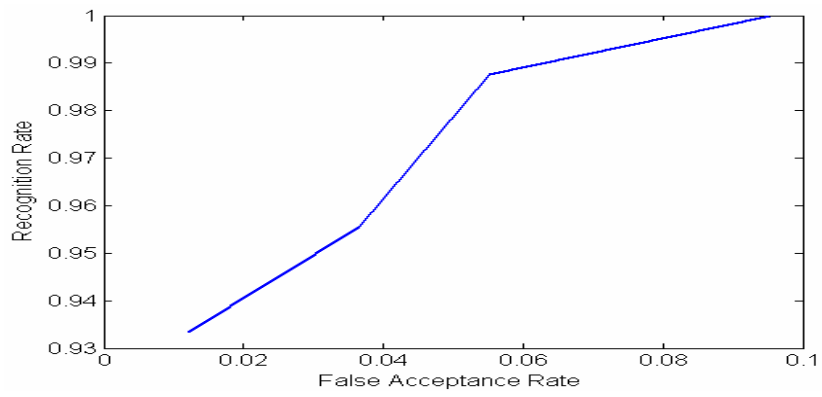

Fig. 11. System performance evaluation using ROC curve

\section{Conclusion and Future Work}

We proposed a personal identification system using palm vein biometrics to detect the liveness of a person. A low cost CCD camera and a set of infrared light source are used to capture the infrared palm images. A subimage is extracted by locating ROI in terms of image registration. The vein within ROI is used as biometric features to do recognition. The experimental results demonstrate that the recognition rate of our system is fine but not good enough to be a real system. At present, our capture device is very sensitive to the outside lights. The outside lights can affect the inside infrared light source so that some images have very poor quality. If the capture device can be improved, the system performance should be better. Further, our database is too small to be convincible. More data are required to be collected for the evaluation of our system.

\section{References}

[1] Lin, C.L., Fan, K.C.: Biometric Verification Using Thermal Images of Palm-Dorsa Vein Patterns. IEEE Transactions on Circuits and Systems for Video Technology 14(2), 199-213 (2004)

[2] Fan, K.C., Lin, C.L: The Using of Thermal Images of Palm-dorsa Vein-patterns for Biometric Verification.In: IEEE ICPR, IEEE Computer Society Press, Los Alamitos (2004)

[3] Yan, K.W., Zhang, Z.Y., Zhuang, D.: Hand Vein Recognition Based on Multi Supplemental Features of Multi-Classifier Fusion Decision. In: IEEE ICMA, IEEE Computer Society Press, Los Alamitos (2006)

[4] Wang, L., Graham, L.: Near- and Far- Infrared Imaging for Vein Pattern Biometrics. In: IEEE ICAVSS, IEEE Computer Society Press, Los Alamitos (2006)

[5] Toh, K., Eng, A.H.L., Choo, Y.S., Cha, Y.L., Yau, W.Y., Low, K.S.: Identity Verification Through Palm Vein and Crease Texture. In: IEEE ICB, pp. 546-553. IEEE Computer Society Press, Los Alamitos (2006)

[6] Zhang, D., Kong, W.K., You, J., Wong, M.: Online Palmprint Identification. IEEE Trans. on Pattern Analysis and Machine Intelligence 25(9), 1041-1050 (2003)

[7] Chaudhuri, S., Chatterjee, S., Katz, N., Nelson, M., Goldbaum, M.: Detection of blood vessels in retinal images using twodimensional matched filters. IEEE Trans. on Medical Imaging 8, 263-269 (1989)

[8] Hoover, A., Kouznetsova, V., Goldbaum, M.: Locating blood vessels in retinal images by piecewise threshold probing of a matched filter response. IEEE Trans. on Medical Imaging 19(3), 203-210 (2000)

[9] Bao, P., Zhang, L.: Noise Reduction for Magnetic Resonance Image via Adaptive Multiscale Products Thresholding. IEEE Trans. on Medical Imaging 22, 1089-1099 (2003)

[10] Bao, P., Zhang, L., Wu, X.L.: Canny Edge Detection Enhancement by Scale Multiplication. IEEE Trans. Pattern Analysis and Machine Intelligence 27(9) (2005)

[11] Li, Q., You, J., Zhang, L., Zhang, D., Bhattacharya, P.: A New Approach to Automated Retinal Vessel Segmentation Using Multiscale Analysis. In: IEEE ICPR, IEEE Computer Society Press, Los Alamitos (2006)

[12] Mallat, S., Zhong, S.: Characterization of signals from multiscale edges. IEEE Trans. Pattern Analysis and Machine Intelligence 14, 710-732 (1992)

[13] Wu, X., Zhang, D., Wang, K.: Palm Line Extraction and Matching for Personal Authentication. IEEE Transactions On Systems, Man, and Cybernetics, Part A 36(5), 978-987 (2006) 